\title{
LACUNARY STRONG A-CONVERGENCE WITH RESPECT TO A MODULUS
}

\author{
TUNAY BILGIN
}

\begin{abstract}
The definition of lacunary strong convergence with respect to a modulus is extended to a definition of lacunary strong A-convergence with respect to a modulus when $A=\left(a_{i k}\right)$ is an infinite matrix of complex numbers. We study some connections between lacunary strong A-convergence with respect to a modulus and lacunary A-statistical convergence.
\end{abstract}

\section{Introduction}

The notion of modulus function was introduced by Nakano [11]. We recall that a modulus $f$ is a function from $[0, \infty)$ to $[0, \infty)$ such that

(i) $f(x)=0$ if and only if $x=0$,

(ii) $f(x+y) \leq f(x)+f(y)$ for $x, y \geq 0$,

(iii) $f$ is increasing and

(iv) $f$ is continuous from the right at 0 . It follows that $f$ must be continuous on $[0, \infty)$.

Connor [2], Esi [3], Kolk [8], Maddox [9], [10], Öztürk and Bilgin [12], Pehlivan and Fisher [13], Ruckle [14] and others used a modulus function to construct sequence spaces.

Following Freedman et al. [4], we call the sequence $\theta=\left(k_{r}\right)$ lacunary if it is an increasing sequence of integers such that $k_{0}=0, h_{r}=k_{r}-k_{r-1} \rightarrow \infty$ as $r \rightarrow \infty$. The intervals determined by $\theta$ will be denoted by $I_{r}=\left(k_{r-1}, k_{r}\right]$ and $q_{r}=k_{r} / k_{r-1}$. These notations will be used throughout the paper. The sequence space of lacunary

1991 Mathematics Subject Classification. 40A05, 40F05.

Key words and phrases. lacunary sequence, modulus function, statistical convergence. 
strongly convergent sequences $N_{\theta}$ was defined by Freedman et al. [4], as follows:

$$
N_{\theta}=\left\{x=\left(x_{i}\right): \lim _{r \rightarrow \infty} h_{r}^{-1} \sum_{i \in I_{r}}\left|x_{i}-s\right|=0 \text { for some } s\right\} .
$$

Recently, the concept of lacunary strongly convergence was generalized by Pehlivan and Fisher [13] as below:

$$
N_{\theta}(f)=\left\{x=\left(x_{i}\right): \lim _{r \rightarrow \infty} h_{r}^{-1} \sum_{i \in I_{r}} f\left(\left|x_{i}-s\right|\right)=0 \text { for some } s\right\} .
$$

Let $A=\left(a_{i k}\right)$ be an infinite matrix of complex numbers. We write $A x=$ $\left(A_{i}(x)\right)$ if $A_{i}(x)=\sum_{k=1}^{\infty} a_{i k} x_{k}$ converges for each $i$.

The purpose of this paper is to introduce and study a concept of lacunary strong A-convergence with respect to a modulus.

\section{2. $N_{\theta}(A, f)$ Convergence}

Definition. Let $A=\left(a_{i k}\right)$ be an infinite matrix of complex numbers and $f$ be a modulus. We define

$$
\begin{gathered}
N_{\theta}(A, f)=\left\{x=\left(x_{i}\right): \lim _{r \rightarrow \infty} h_{r}^{-1} \sum_{i \in I_{r}} f\left(\left|A_{i}(x)-s\right|\right)=0 \text { for some } s\right\}, \\
N_{\theta}^{0}(A, f)=\left\{x=\left(x_{i}\right): \lim _{r \rightarrow \infty} h_{r}^{-1} \sum_{i \in I_{r}} f\left(\left|A_{i}(x)\right|\right)=0\right\}
\end{gathered}
$$

A sequence $x=\left(x_{k}\right)$ is said to be lacunary strong A-convergent to a number $s$ with respect to a modulus if there is a complex number $s$ such that $x \in N_{\theta}(A, f)$. Note that, if we put $f(x)=x$, then $N_{\theta}(A, f)=N_{\theta}(A)$ and $N_{\theta}^{0}(A, f)=N_{\theta}^{0}(A)$. If $x \in N_{\theta}(A)$, we say that $x$ is lacunary strong A-convergent to $s$. If $x$ is lacunary strong A-convergent to the value $s$ with respect to a modulus $f$, then we write $x_{i} \rightarrow$ $s\left(N_{\theta}(A, f)\right)$. If $A=I$ unit matrix, we write $N_{\theta}(f)$ and $N_{\theta}^{0}(f)$ for $N_{\theta}(A, f)$ and $N_{\theta}^{0}(A, f)$, respectively. Hence $N_{\theta}(f)$ is the same as the space $N_{\theta}(f)$ of Pehlivan and Fisher [13].

$N_{\theta}(A, f)$ and $N_{\theta}^{0}(A, f)$ are linear spaces. We consider only $N_{\theta}^{0}(A, f)$. Suppose that $x, y \in N_{\theta}^{0}(A, f)$ and $a, b$ are in $C$, the complex numbers. Then there exist integers 40 
$T_{a}$ and $T_{b}$ such that $|a| \leq T_{a}$ and $|b| \leq T_{b}$. We therefore have

$$
h_{r}^{-1} \sum_{i \in I_{r}} f\left(\left|a A_{i}(x)+b A_{i}(y)\right|\right) \leq T_{a} h_{r}^{-1} \sum_{i \in I_{r}} f\left(\left|A_{i}(x)\right|\right)+T_{b} h_{r}^{-1} \sum_{i \in I_{r}} f\left(\left|A_{i}(y)\right|\right) .
$$

This implies that $a x+b y \in N_{\theta}^{0}(A, f)$.

Now we give relation between lacunary strong A-convergence and lacunary strong A-convergence with respect to a modulus.

Theorem 1. Let $f$ be any modulus. Then $N_{\theta}(A) \subseteq N_{\theta}(A, f)$ and $N_{\theta}^{0}(A) \subseteq$ $N_{\theta}^{0}(A, f)$.

Proof. We consider $N_{\theta}(A) \subseteq N_{\theta}(A, f)$ only. Let $x \in N_{\theta}(A)$ and $\varepsilon \succ 0$. We choose $0<\delta<1$ such that $f(u)<\varepsilon$ for every $u$ with $0 \leq u \leq \delta$. We can write

$$
h_{r}^{-1} \sum_{i \in I_{r}} f\left(\left|A_{i}(x)-s\right|\right)=h_{r}^{-1} \sum_{1} f\left(\left|A_{i}(x)-s\right|\right)+h_{r}^{-1} \sum_{2} f\left(\left|A_{i}(x)-s\right|\right)
$$

where the first summation is over $\left|A_{i}(x)-s\right| \leq \delta$ and the second over $\left|A_{i}(x)-s\right| \succ \delta$. By definition of $f$, we have

$$
h_{r}^{-1} \sum_{i \in I_{r}} f\left(\left|A_{i}(x)-s\right|\right) \leq \varepsilon+2 f(1) \delta^{-1} h_{r}^{-1} \sum_{i \in I_{r}}\left|A_{i}(x)-s\right| .
$$

Therefore $x \in N_{\theta}(A, f)$.

Theorem 2. Let $f$ be any modulus. If $\lim _{t \rightarrow \infty} \frac{f(t)}{t}=\beta \succ 0$, then $N_{\theta}(A)=$ $N_{\theta}(A, f)$.

Proof. If $\lim _{t \rightarrow \infty} \frac{f(t)}{t}=\beta \succ 0$, then $f(t) \geq \beta t$ for all $t \succ 0$. Let $x \in N_{\theta}(A, f)$. Clearly,

$$
h_{r}^{-1} \sum_{i \in I_{r}} f\left(\left|A_{i}(x)-s\right|\right) \geq h_{r}^{-1} \sum_{i \in I_{r}} \beta\left|A_{i}(x)-s\right|=\beta h_{r}^{-1} \sum_{i \in I}\left|A_{i}(x)-s\right|,
$$

therefore $x \in N_{\theta}(A)$. By using Theorem 1 the proof is complete.

We now give an example to show that $N_{\theta}(A) \neq N_{\theta}(A, f)$ in the case when $\beta=0$. Consider $A=I$ and the modulus $f(x)=\sqrt{x}$. In the case $\beta=0$, define $x_{i}$ to be $h_{r}$ at the first term in $I_{r}$ for every $r$ and $x_{i}=0$ otherwise. Then we have

$$
h_{r}^{-1} \sum_{i \in I_{r}} f\left(\left|A_{i}(x)\right|\right)=h_{r}^{-1} \sum_{i \in I_{r}} \sqrt{\left|x_{i}\right|}=h_{r}^{-1} \sqrt{\left|h_{r}\right|} \rightarrow 0 \text { as } r \rightarrow \infty
$$

and so $x \in N_{\theta}(A, f)$. But $h_{r}^{-1} \sum_{i \in I_{r}}\left|A_{i}(x)\right|=h_{r}^{-1} \sum_{i \in I_{r}}\left|x_{i}\right|=h_{r}^{-1} h_{r} \rightarrow 1$ as $r \rightarrow \infty$ and so $x \notin N_{\theta}(A)$.

Theorem 3. Let $f$ be any modulus. Then 
(i) For $\liminf q_{r} \succ 1$ we have $w(A, f) \subseteq N_{\theta}(A, f)$.

(ii) For $\lim \sup q_{r} \prec \infty$ we have $N_{\theta}(A, f) \subseteq w(A, f)$.

(iii) $w(A, f)=N_{\theta}(A, f)$ is $1 \succ \liminf _{r} q_{r} \leq \lim \sup _{r} q_{r} \prec \infty$,

where $w(A, f)=\left\{x=\left(x_{i}\right): \lim _{n \rightarrow \infty} n^{-1} \sum_{i=1}^{n} f\left(\left|A_{i}(x)-s\right|\right)=0\right.$ for some $\left.s\right\}$ (see, Esi $[3])$.

Proof. (i) Let $x \in w(A, f)$ and $\liminf q_{r} \succ 1$. There exist $\delta \succ 0$ such that $q_{r}=\left(k_{r} / k_{r-1}\right) \geq 1+\delta$ for sufficiently large $r$. We have, for sufficiently large $r$, that $\left(h_{r} / k_{r}\right) \geq \delta /(1+\delta)$ and $\left(k_{r} / h_{r}\right) \leq(1+\delta) / \delta$. Then

$$
\begin{aligned}
k_{r}^{-1} \sum_{i-1}^{k_{r}} f\left(\left|A_{i}(x)-s\right|\right) & \geq k_{r}^{-1} \sum_{i \in I_{r}} f\left(\left|A_{i}(x)-s\right|\right) \\
& =\left(h_{r} / k_{r}\right) h_{r}^{-1} \sum_{i \in I_{r}} f\left(\left|A_{i}(x)-s\right|\right) \\
& \geq \delta /(1+\delta) h_{r}^{-1} \sum_{i \in I_{r}} f\left(\left|A_{i}(x)-s\right|\right)
\end{aligned}
$$

which yields that $x \in N_{\theta}(A, f)$.

(ii) If $\lim \sup q_{r} \prec \infty$ then there exists $K \succ 0$ such that $q_{r} \prec K$ for every $r$. Now suppose that $x \in N_{\theta}(A, f)$ and $\varepsilon \succ 0$. There exists $m_{0}$ such that for every $m \geq m_{0}$,

$$
H_{m}=h_{m}^{-1} \sum_{i \in I_{m}} f\left(\left|A_{i}(x)-x\right|\right) \prec \varepsilon .
$$

We can also find $T \succ 0$ such that $H_{m} \leq T$ for all $m$. Let $n$ be any integer with $k_{r} \geq n \succ k_{r-1}$. Now write

$$
\begin{gathered}
n^{-1} \sum_{i=1}^{n} f\left(\left|A_{i}(x)-s\right|\right) \leq k_{r}^{-1} \sum_{i=1}^{k_{r}} f\left(\left|A_{i}(x)-s\right|\right) \\
=k_{r-1}^{-1}\left(\sum_{m=1}^{m_{0}}+\sum_{m=m_{0}+1}^{k_{r}}\right) \sum_{i \in I_{m}} f\left(\left|A_{i}(x)-s\right|\right) \\
=k_{r-1}^{-1} \sum_{m=1}^{m_{0}} \sum_{i \in I_{m}} f\left(\left|A_{i}(x)-s\right|\right)+k_{r-1}^{-1} \sum_{m=m_{0}+1}^{k_{r}} \sum_{i \in I_{m}} f\left(\left|A_{i}(x)-s\right|\right) \\
\leq k_{r-1}^{-1} \sum_{m=1}^{m_{0}} \sum_{i \in I_{m}} f\left(\left|A_{i}(x)-s\right|\right)+\varepsilon\left(k_{r}-k_{m_{0}}\right) k_{r-1}^{-1} \\
=k_{r-1}^{-1}\left(h_{1} H_{1}+h_{2} H_{2}+\cdots+h_{m_{0}} H_{m_{0}}\right)+\varepsilon\left(k_{r}-k_{m_{0}}\right) k_{r-1}^{-1} \\
\leq k_{r-1}^{-1}\left(\sup _{1 \leq i \leq m_{0}} H_{i} k_{m_{0}}\right)+\varepsilon K \prec k_{r-1}^{-1} k_{m_{0}} T+\varepsilon K
\end{gathered}
$$


from which we deduce that $x \in w(A, f)$. (iii) follows from (i) and (ii).

The next result follows from Theorem 2 and 3.

Theorem 4. Let $f$ be any modulus. If $\lim _{t \rightarrow \infty} \frac{f(t)}{t}=\beta \succ 0$ and $l \prec$ $\liminf _{r} q_{r} \leq \lim \sup _{r} q_{r} \prec \infty$, then $N_{\theta}(A)=w(A, f)$.

\section{Lacunary A-statistical convergence}

The notation of statistical convergence was given in earlier works [1], [4], [6], [15] and [16]. Recently, Fridy and Orhan [7] introduced the concept of lacunary statistical convergence:

Let $\theta$ be a lacunary sequence. Then a sequence $x=\left(x_{k}\right)$ is said to be lacunary statistically convergent to a number $s$ if for every $\varepsilon \succ 0, \lim _{r \rightarrow \infty} h_{r}^{-1}\left|K_{\theta}(\varepsilon)\right|=0$, where $\left|K_{\theta}(\varepsilon)\right|$ denotes the number of elements in $K_{\theta}(\varepsilon)=\left\{i \in I_{r}:\left|x_{i}-s\right| \geq \varepsilon\right\}$. The set of all lacunary statistical convergent sequences is denoted by $S_{\theta}$.

Let $A=\left(a_{i k}\right)$ be an infinire matrix of complex numbers. Then a sequence $x=\left(x_{k}\right)$ is said to be lacunary A-statistically convergent to a number $s$ if for every $\varepsilon \succ 0, \lim _{r \rightarrow \infty} h_{r}^{-1}\left|K A_{\theta}(\varepsilon)\right|=0$, where $\left|K A_{\theta}(\varepsilon)\right|$ denotes the number of element in $K A_{\theta}(\varepsilon)=\left\{i \in I:\left|A_{i}(x)-s\right| \geq \varepsilon\right\}$. The set of all lacunary A-statistical convergent sequences is denoted by $S_{\theta}(A)$.

The following Theorem gives the relation between of the lacunary A-statistical convergence and lacunary strongly A-convergence.

Let $I_{r}^{1}=\left\{i \in I_{r}:\left|A_{i}(x)-s\right| \geq \varepsilon\right\}=K A_{\theta}(\varepsilon)$ and $I_{r}^{2}=\left\{i \in I_{r}:\left|A_{i}(x)-s\right| \prec\right.$ $\varepsilon\}$.

Theorem 5. Let $A$ be a limitation method, then

(i) $x_{i} \rightarrow s\left(N_{\theta}(A)\right)$ implies $x_{i} \rightarrow s\left(S_{\theta}(A)\right)$.

(ii) $x$ is bounded and $x_{i} \rightarrow s\left(S_{\theta}(A)\right)$ implys $x_{i} \rightarrow s\left(N_{\theta}(A)\right)$.

(iii) $S_{\theta}(A)=N_{\theta}(A)$ is $x$ is bounded.

Proof. (i) If $\varepsilon \succ 0$ and $x_{i} \rightarrow s\left(N_{\theta}(A)\right)$ we can write

$$
h_{r}^{-1} \sum_{i \in I_{r}}\left|A_{i}(x)-s\right| \geq h_{r}^{-1}\left|K A_{\theta}(\varepsilon)\right| \varepsilon
$$

It follows that $x_{i} \rightarrow s\left(S_{\theta}(A)\right)$. Note that in this part of the proof we do not need the limitation method of $A$. 
(ii) Suppose that $x$ is lacunary A-statistical convergent to $s$. Since $x$ is bounded and $A$ is limitation method, there is a constant $T>0$ such that $\left|A_{i}(x)-s\right| \leq T$ for all $i$. Therefore we have, for every $\varepsilon \succ 0$, that

$h_{r}^{-1} \sum_{i \in I_{r}}\left|A_{i}(x)-s\right| \leq h_{r}^{-1} \sum_{i \in I_{r}^{1}}\left|A_{i}(x)-s\right|+h_{r}^{-1} \sum_{i \in I_{r}^{2}}\left|A_{i}(x)-s\right| \leq T h_{r}^{-1}\left|K A_{\theta}(\varepsilon)\right|+\varepsilon$.

Taking the limit as $\varepsilon \rightarrow 0$, the result follows. (iii) follows from (i) and (ii).

Now we give the relation between of the lacunary A-statistical convergence and lacunary strongly A-convergence with respect to modulus.

Theorem 6. (i) For any modulus $f, x_{i} \rightarrow s\left(N_{\theta}(A, f)\right)$ implies $x_{i} \rightarrow$ $s\left(S_{\theta}(A)\right)$.

(ii) $f$ is bounded and $x_{i} \rightarrow s\left(S_{\theta}(A)\right)$ imply $x_{i} \rightarrow s\left(N_{\theta}(A, f)\right)$.

(iii) $S_{\theta}(A)=N_{\theta}(A, f)$ if $f$ is bounded.

Proof. (i) Let $f$ be any modulus. If $\varepsilon \succ 0$ and $x_{i} \rightarrow s\left(N_{\theta}(A, f)\right)$ we can write

$$
h_{r}^{-1} \sum_{i \in I_{r}} f\left(\left|A_{i}(x)-s\right|\right) \geq h_{r}^{-1} \sum_{i \in I_{r}^{1}} f\left(\left|A_{i}(x)-s\right|\right) \succ h_{r}^{-1}\left|K A_{\theta}(\varepsilon)\right| f(\varepsilon) .
$$

It follows that $x_{i} \rightarrow s\left(S_{\theta}(A)\right)$.

(ii) Suppose that $f$ is bounded. Since $f$ is bounded, there exists an integer $T$ such that $f(x) \leq T$ for all $x \geq 0$. We see that

$$
\begin{aligned}
h_{r}^{-1} \sum_{i \in I_{r}} f\left(\left|A_{i}(x)-s\right|\right) & \leq h_{r}^{-1} \sum_{i \in I_{r}^{1}} f\left(\left|A_{i}(x)-s\right|\right)+h_{r}^{-1} \sum_{i \in I_{r}^{2}} f\left(\left|A_{i}(x)-s\right|\right) \\
& \leq T h_{r}^{-1}\left|K A_{\theta}(\varepsilon)\right|+f(\varepsilon) .
\end{aligned}
$$

Since $f$ is continuous and $x_{i} \rightarrow s\left(S_{\theta}(A)\right)$, it follows from $\varepsilon \rightarrow 0$ that $x_{i} \rightarrow$ $s\left(N_{\theta}(A, f)\right)$. (ii) follows from (i) and (ii).

As an example to show that $S_{\theta}(A) \neq N_{\theta}(A, f)$ when $f$ is unbounded, consider $A=I$. Since $f$ is unbounded, there exists a positive sequence $0 \prec y_{1} \prec y_{2} \prec \ldots$ such that $f\left(y_{i}\right) \geq h_{i}$. Define the sequence $x=\left(x_{i}\right)$ by putting $x_{k_{i}}=y_{i}$ for $i=1,2, \ldots$ and $x_{i}=0$ otherwise. We have $x \in S_{\theta}(A)$, but $x \notin N_{\theta}(A, f)$.

Finally we consider the case when $x_{k} \rightarrow s$ implies $x_{k} \rightarrow s\left(N_{\theta}(A, f)\right)$.

Lemma 7. ([6]) If liminf $q_{r} \succ 1$ then $x_{i} \rightarrow s(S)$ implies $x_{i} \rightarrow s\left(S_{\theta}\right)$. 
Theorem 8. Let $\liminf q_{r} \succ 1, A$ is regular and $f$ is bounded. Then $x_{i} \rightarrow s$ implies $x_{i} \rightarrow s\left(N_{\theta}(A, f)\right)$.

Proof. Let $x_{i} \rightarrow s$. By regularity of $A$ and definition of statistical convergence we have $A_{i}(x) \rightarrow s(S)$. Since $\liminf q_{r} \succ 1$ it follows lemma 7 that $A_{i}(x) \rightarrow s\left(S_{\theta}\right)$ i.e. $x_{i} \rightarrow s\left(S_{\theta}(A)\right)$. Thus, using Theorem 6 , we have $x_{i} \rightarrow s\left(N_{\theta}(A, f)\right)$.

\section{References}

[1] Connor, J.S., The statisical and strong p-Cesaro convergence of sequences, Analysis, 8(1988), 47-63.

[2] Connor, J.S., On strong matrix summalibity with respect to a modulus and statistical convergence, Canad. Math. Bull. 32(1989), 194-198.

[3] Esi, A., The A-statistical and strongly (A-p)-Cesaro convergence of sequences, Pure and Appl. Math. Sci., Vol.XLIII, No.1-2(1996), 89-93.

[4] Fast, H., Sur la convergence statistique, Colloq. Math. 2(1951), 241-244.

[5] Freedman, A.R., Sember, J.J., Raphel, M., Some Cesaro-type summability spaces, Proc. London Math. Soc. 37(3)(1978), 508-520.

[6] Frydy, J., On statistical convergence, Analysis, 5(1985), 301-313.

[7] Frydy, J., Orhanc, C., Lacunary statistical convergence, Pacific J. Math. 160(1993), $45-51$.

[8] Kolk, E., On strong boundedness and summability with respect to a sequence moduli, Tartu Ül. Toimetised 960(1993), 41-50.

[9] Maddox, I.J., Sequence spaces defined by a modulus, Math. Proc. Camb. Phil. Soc. 100(1986), 161-166.

[10] Maddox, I.J., Inclusion between FK spaces and Kuttner's theorem, Math. Proc. Camb. Phil. Soc., 101(1987), 523-527.

[11] Nakano, H., Concave modulars, J. Math. Soc. Japan, 5(1953), 29-49.

[12] Öztürk, E., Bilgin, T., Strongly summable sequence spaces defined by a modulus, Indian J. Pure Appl. Math., 25(6)(1994), 621-625.

[13] Pehlivan, S., Fisher, B., On some sequence spaces, Indian J. Pure Appl. Math. 25(10)(1994), 1067-1071.

[14] Ruckle, W.H., FK spaces in which the sequence of coordinate vectors is bounded, Canad. J. Math., 25(1973), 973-978.

[15] Salat, T., On statistically convergent sequences of real numbers, Math. Slovaca, 2(1980), $139-150$.

[16] Schoenberg, I.J., The integrability of certain functions and related summability methods, Amer. Math. Monthly, 66(1959), 261-375.

Department of Mathematics, University of 100.Yil, VAn Turkey 